\title{
Investigating optimal education regarding the low FODMAP diet in functional bowel disorders: a feasibility randomised controlled trial of leaflet vs mobile application vs dietetic consultation
}

\author{
Eirini Dimidi ${ }^{1}$, Kevin Whelan ${ }^{1}$ and Miranda CE Lomer ${ }^{1,2}$ \\ ${ }^{1}$ King's College London, Department of Nutritional Sciences, London, United Kingdom and \\ ${ }^{2}$ Guy's and St Thomas' NHS Foundation Trust, Department of Nutrition and Dietetics, London, United Kingdom
}

A diet low in fermentable carbohydrates (FODMAPs) improves symptoms of irritable bowel syndrome (IBS) ${ }^{(1)}$. Although it is recommended patients receive advice on a low FODMAP diet by a dietitian ${ }^{(2)}$, this is labour-intensive and costly, highlighting the need for alternative effective education delivery methods. This study aimed to establish the feasibility of undertaking a trial that assesses the clinical and cost effectiveness of different education delivery methods (leaflets vs mobile application vs one-to-one consultation with a dietitian) of the low FODMAP diet.

Patients diagnosed with IBS, functional bloating or functional diarrhoea based upon the Rome IV criteria were recruited in this parallel group, 4-week, feasibility randomised controlled trial. Patients were randomised 1:1:1 to receive information about the low FODMAP diet using leaflets (leaflet), mobile application (app), or one-to-one consultation with a dietitian (dietitian). Feasibility outcomes included recruitment and retention rates, and acceptability of the interventions. Symptoms were assessed using the validated "adequate symptom relief" question and IBS-Symptom Scoring System (IBS-SSS). Analysis of variance was used to analyse continuous outcomes, and chi-squared test for categorical outcomes.

Fifty-one patients were randomised. Overall, recruitment rate was 2.4 patients/month and retention rates were $18 / 19(95 \%)$ in the leaflet, $16 / 17(94 \%)$ in the app and $14 / 15(93 \%)$ in the dietitian group. A higher proportion of patients in the dietitian group (64\%) strongly agreed they were "able to implement the low FODMAP diet" compared with the app (13\%) and leaflet groups (6\%; $\mathrm{p}=0.008)$. More patients reported "adequate symptom relief" in the dietitian group $(80 \%)$ compared to those in the leaflet group at follow up $(39 \%, p=0.026)$, but not compared to the app $(63 \%)$. Although there was no significant difference in total IBS-SSS scores among the groups $(\mathrm{p}=0.438)$, change in IBS-SSS from baseline was significantly greater in the dietitian group $(-153 \pm 90)$ compared to the leaflet group $(-90 \pm 56 ; \mathrm{p}=0.048)$, but not compared to the app $(-120 \pm 62)$. More patients in the leaflet group $(83 \%)$ reported they would have preferred to have received a different education method compared with those in the app (44\%) and dietitian groups $(14 \% ; \mathrm{p}<0.001)$.

To conclude, this feasibility study confirms that an adequately powered trial of the most clinically and cost-effective education delivery method for the low FODMAP diet is required. Preliminary evidence suggests that delivering low FODMAP diet advice through a one-to-one consultation with a dietitian improves functional bowel symptoms compared to leaflets, but not compared to the app.

1. Staudacher and Whelan. (2017) Gut 66(8), 1517-27.

2. O'Keeffe and Lomer. (2017) J Gastroenterol Hepatol 32(1), 23-6. 